\title{
A New High Energy-resolution Soft-X-ray Spectrometer for A Transmission Electron Microscope
}

\author{
M. Terauchi and M. Koike*
}

Institute of Multidisciplinary Research for Advanced Materials, Tohoku University, 2-1-1 katahira, Aoba-ku, Sendai 980-8577, JAPAN

* Advanced Photon Research Center, Kansai Research Establishment, JAERI, 8-1 Umemidai, Kizu-cho, Souraku-gun, Kyoto 619-0215, JAPAN

We have been developing a high energy-resolution soft-X-ray spectrometer for a transmission electron microscope to obtain the information of the density of states (DOS) of the valence band (occupied states) from identified small specimen areas [1,2].

The spectrometer $\left(2^{\text {nd }}\right.$ version [3]) was composed of varied-line-spacing (VLS) gratings and a CCD detector. An energy range from $60 \mathrm{eV}$ to $1200 \mathrm{eV}$ was accessible by using two VLS gratings, which have line densities of 1200 and 2400 lines $/ \mathrm{mm}$. The size of the CCD detector was $27.6 \times 27.6 \mathrm{~mm}^{2}(2 \mathrm{kx} 2 \mathrm{k})$, which corresponds to a collection angle of $6.5 \times 10^{-4} \mathrm{sr}$. By introducing X-ray reflection flat mirrors, spectral intensity was improved by 2.2 times. Thus, the effective collection angle of the spectrometer was $1.4 \times 10^{-3} \mathrm{sr}$. Energy resolutions of this spectrometer for Si $L$-emission $(\sim 100 \mathrm{eV}), \mathrm{B} K$-emission $(\sim 180 \mathrm{eV})$ and $\mathrm{Cu} L$-emission $(\sim 930$ $\mathrm{eV}$ ) were evaluated to be $0.1,0.4$ and $1.4 \mathrm{eV}$, respectively. This $2^{\text {nd }}$ spectrometer successfully obtained the DOS of the valence band from specified small specimen areas of $h, c, w-\mathrm{BN}$, $\alpha$, $\beta$-boron, $\mathrm{Si}$, carbon allotropes and quasicrystals. However, the collection angle and the energy resolution of higher energy region were not enough.

We have designed and produced new X-ray reflection mirrors and a new VLS grating. The new X-ray reflection curved mirrors were designed to improve the solid angle of the spectrometer by about two times bigger than that of the $2^{\text {nd }}$ spectrometer. The mirrors were produce by a sputtering of tungsten on substrates. The tungsten layer is about $100 \mathrm{~nm}$ in thickness. The new grating was designed to have a focal distance of about $50 \mathrm{~cm}$, which is about two times larger than that of the previous grating. Thus, the dispersion of the new one is about two times larger than before. Figure 1 shows energy dispersions (energy / CCD channel) of the previous gratings of $1200 \mathrm{l} / \mathrm{mm}, 2400 \mathrm{l} / \mathrm{mm}$ and the newly designed grating of $2400 \mathrm{l} / \mathrm{mm}$. Aberration is smaller than one CCD pixel size. Thus, an energy resolution of about $0.7 \mathrm{eV}$ is expected for X-ray energy of about $1000 \mathrm{eV}$. Figure 2 shows a photo of the spectrometer $\left(3^{\text {rd }}\right.$ version) attached to a transmission electron microscope of JEM2000FX. The design and the performance of the spectrometer will be presented.

[1] M. Terauchi, H. Yamamoto and M. Tanaka: J. Electron Microscopy, $\underline{50}$ (2001) 101.

[2] M. Terauchi, H. Yamamoto and M. Tanaka: Microsc. Microanal., 7 suppl.2 (2001) 228. 
[3] M. Terauchi and M. Kawana: Microsc. Microanal., $\underline{8}$ suppl.2 (2002) 644.

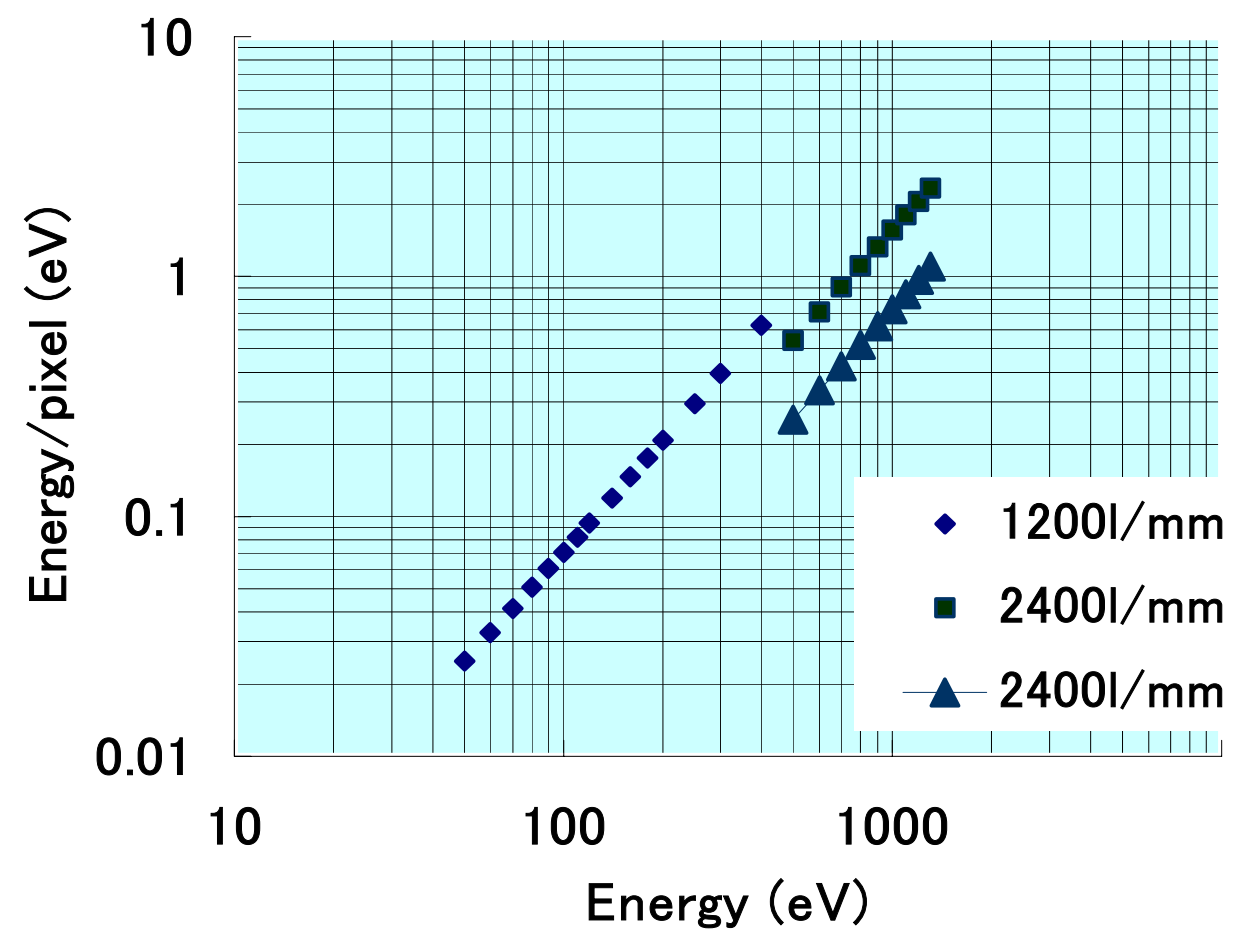

FIG.1 Energy dispersions (energy / CCD pixel) of the gratings of $12001 / \mathrm{mm}(\bullet), 2400$ $1 / \mathrm{mm}(\boldsymbol{\square})$ already used and the newly designed grating of $24001 / \mathrm{mm}(\boldsymbol{\Delta})$.

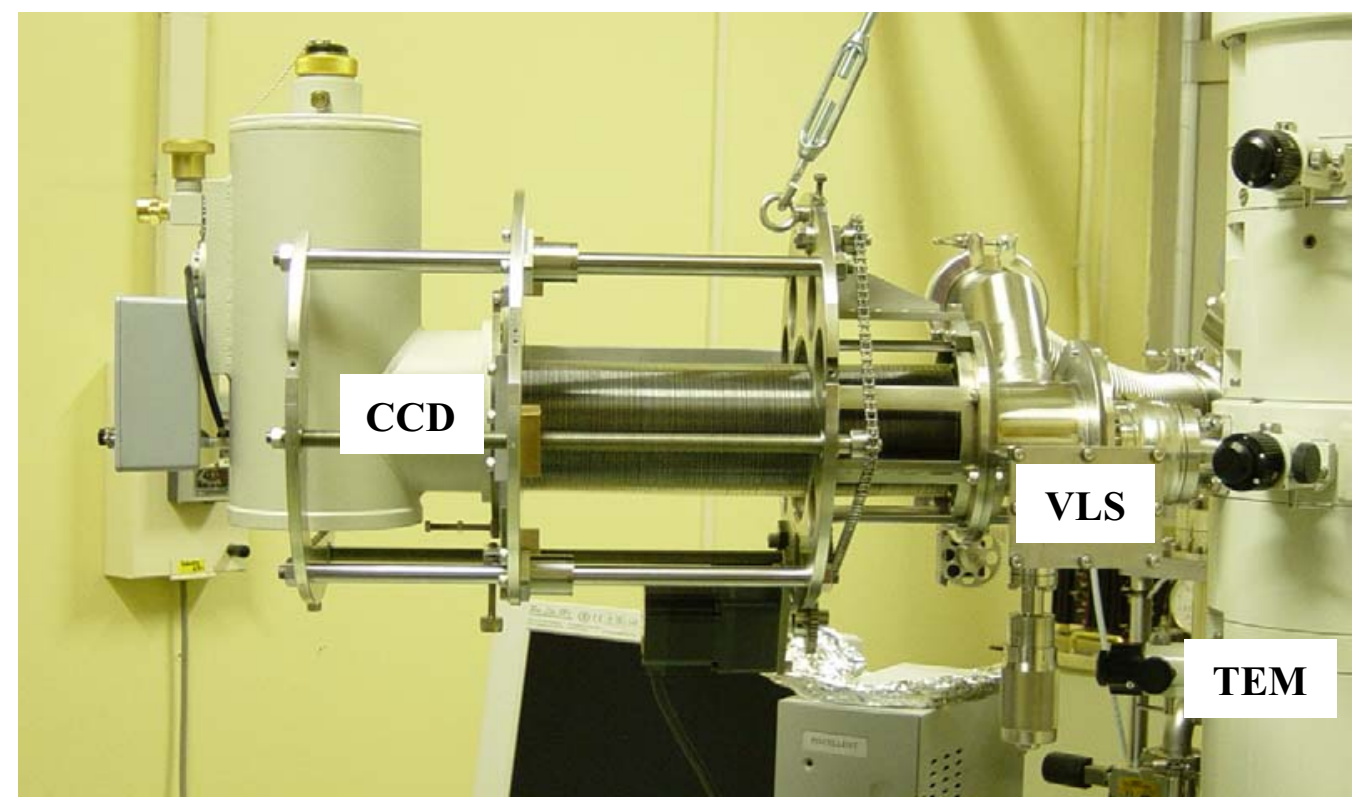

FIG.2 Photo of the spectrometer ( $3^{\text {rd }}$ version) attached to a transmission electron microscope of JEM2000FX. 